\title{
Postoperative management and acute complications after lung transplantation
}

\author{
Osama Haddad, Mathew Thomas \\ Department of Cardiothoracic Surgery, Mayo Clinic, Jacksonville, FL, USA \\ Contributions: (I) Conception and design: Both authors; (II) Administrative support: None; (III) Provision of study materials or patients: None; (IV) \\ Collection and assembly of data: Both authors; (V) Data analysis and interpretation: Both authors; (VI) Manuscript writing: Both authors; (VII) Final \\ approval of manuscript: Both authors. \\ Correspondence to: Mathew Thomas. 4500 San Pablo Rd S, Jacksonville, FL 32224, USA. Email: Thomas.Mathew@mayo.edu.
}

\begin{abstract}
Lung transplantation is an established therapy for select patients with end-stage pulmonary disease, offering prolonged survival and significant improvement in recipient's quality of life. The postoperative care of lung transplant recipients is quite complex and deals with the prevention and management of a wide spectrum of immediate post-operative complications. Since these complications occur frequently and are associated with poor outcomes including increased short and long-term morbidity and mortality, it is extremely important to recognize and manage them early. It is worth noting that the post-operative critical care of lung transplant recipients is multidisciplinary in nature and presents with evolving clinical scenarios that require frequent changes in treatment plans. In addition to the medical and surgical teams, allied health providers like respiratory, physical and nutritional therapists play a pivotal role in the management of patients. Close collaboration and communication between the different teams will eventually translate into a better outcomes and survival. In this review, the first part will highlight the important aspects of the immediate care of the lung transplant recipients following surgery including monitoring, ventilator and hemodynamic management, pain control, immunosuppression and the prevention of infection in addition to other nuances. The second part of the review will discuss the diagnosis and management of acute medical [primary graft dysfunction (PGD), acute allograft rejection] and surgical complications such as those related to the airways, vascular anastomosis or pleural space.
\end{abstract}

Keywords: Lung transplantation; complications; post-operative care

Received: 03 December 2020. Accepted: 09 April 2021.

doi: $10.21037 /$ ccts-20-182

View this article at: http://dx.doi.org/10.21037/ccts-20-182

\section{Introduction}

Despite the tremendous advancements in surgical techniques and immediate post-operative care, the 3 -month mortality rate after lung transplantation is still high at $9 \%$ (1). Recipients who survive to 1 year after primary transplant have been shown to have a conditional median survival of 7.9 years (1). Hence the optimization of immediate post-operative care and the recognition and management of early complications have a critical role in improving long-term survival.

\section{Immediate post-operative care}

\section{Monitoring}

Post-operative monitoring continues from the operating room with both invasive and non-invasive monitoring techniques. Routine invasive monitoring involves the use of arterial pressure lines, pulmonary artery (PA) catheter and urinary bladder catheter. Non-invasive monitoring includes continuous electrocardiogram, pulse oximetry and capnometry. Bedside echocardiography with transthoracic or occasionally transesophageal (TEE) is a useful modality 
to rule out pericardial tamponade or evaluate cardiac function in hemodynamically unstable patients who do not respond to resuscitation. Additionally, TEE is used to assess the PA anastomosis in cases of persistent or severe unexplained pulmonary hypertension, or to assess the pulmonary vein $(\mathrm{PV})$ anastomosis if there is evidence of localized pulmonary edema to one lung or lobe. Intensive care unit (ICU) monitoring should provide information needed for diagnostic evaluation and planning of care. Since lung recipients tend to have a prolonged ICU stay, special care should be taken to prevent indwelling catheter and line infections $(2,3)$. Catheters and invasive lines that do not provide further essential information should be removed promptly once the patient is stable.

\section{Hemodynamic support}

The goal of hemodynamic management is to ensure adequate blood pressure and optimal organ perfusion. End-organ perfusion is assessed by hourly urine output in addition to venous saturation $\left(\mathrm{SvO}_{2}\right)$, periodic serum lactate and serum creatinine levels. Significant fluid shifts between intravascular and extravascular spaces occur after lung transplantation and it is important to adjudicate fluid administration based on this. The recently transplanted lung allograft is susceptible to reperfusion injury from fluid overload, especially in the presence of a hyperdynamic right ventricle. Hence, if vasopressor therapy is needed, norepinephrine or vasopressin is preferred instead of inotropes that increase cardiac contractility. Low $\mathrm{SvO}_{2}$ with hemodynamic instability should be evaluated with an echocardiogram to rule out thoracic compartment syndrome or cardiac tamponade, and to evaluate right and left heart functions. The preferred agent for inotropic support is lowdose dobutamine $(2-3 \mathrm{mcg} / \mathrm{kg} / \mathrm{min}$ ). Milrinone should be used cautiously as it may cause vasodilation and arrhythmia. Epinephrine may cause pulmonary edema secondary to increased adrenergic activity (4).

\section{Ventilator management}

The success of protective lung ventilation in acute respiratory distress syndrome has led to wide adoption of this strategy in ventilating lung recipients immediately after transplantation. Low tidal volume $(<6 \mathrm{~mL} / \mathrm{kg}$ of ideal body weight, low positive end-expiratory pressure (PEEP) $\left(5-10 \mathrm{~cm} \mathrm{H}_{2} \mathrm{O}\right)$ and adjusting the respiratory rate to control minute ventilation are maneuvers used to provide adequate oxygenation and correct respiratory acidosis. Lung protective ventilation is aimed at reducing primary graft dysfunction (PGD) and ventilator-induced lung injury. With adequate amount of PEEP (up to $10 \mathrm{~cm} \mathrm{H}_{2} \mathrm{O}$ ) it is uncommon for the protective lung ventilation to cause atelectasis. The duration of ventilation may vary according to the individual patient; most uncomplicated cases can be extubated within hours to few days (5), while patients on extra-corporeal membrane oxygenation (ECMO) support may need to remain ventilated until it can be successfully discontinued. Extubation criteria include hemodynamic stability, ability to protect airway, $\mathrm{FiO}_{2}<50 \%$, PEEP of $5 \mathrm{~cm}$ $\mathrm{H}_{2} \mathrm{O}$, Nitric Oxide $(\mathrm{NO})<10 \mathrm{ppm}$, respiratory rate $<20 / \mathrm{min}$ and minute ventilation $<10 \mathrm{~mL} / \mathrm{kg}$. For patients who fail to progress, early tracheostomy will facilitate weaning of sedation, pulmonary toilet and ventilator weaning (6).

The differential diagnoses of post-operative hypoxemia and hypercapnia are broad. General causes include fluid overload, heart failure, atelectasis, pneumonia, pleural effusion, pneumothorax, venous thromboembolism, bronchoconstriction and pericardial tamponade. Specific causes related to lung transplantation include PGD and anastomotic complications. A plain chest X-ray done at the bedside can help identify atelectasis or lung compression, pleural effusions, hemothorax or pneumothorax. In most instances of hypoxemia and hypercapnia, bronchoscopy is indicated to remove airway debris, blood clots and secretions, and can improve gas exchange. If still hypoxic, more investigation is warranted such as contrastenhanced CT scan to check the pulmonary vasculature, anastomosis and lung parenchyma. If intravenous contrast is contraindicated, a non-contrast CT aided by TEE interrogation of the PVs and perfusion scan can provide diagnostic information.

\section{Special Consideration in single-lung transplant recipients}

Single-lung recipients may have either obstructive [chronic obstructive pulmonary disease (COPD), emphysema] or restrictive (e.g., pulmonary fibrosis) pathology in the native lung. In case of obstructive pathology, the native lung is more compliant, leading to over distension and autoPEEP, while edema in the transplanted lung may cause lower compliance on that side. The incidence of acute hyperinflation in single lung transplants with COPD can be as high as $30 \%$ (7). Ventilator settings should account for the donor height to avoid hyperinflation of the allograft as this may increase the risk of PGD and prolonged intubation (8). 
In patients with obstructive pathology, ventilation strategies include PEEP $\leq 5 \mathrm{~cm} \mathrm{H}_{2} \mathrm{O}$, short inspiratory time, prolonged expiratory time and lower respiratory rate $(8-10 / \mathrm{min})$. Differential (split) lung ventilation through a double lumen endotracheal tube and using with two ventilators is another strategy where protective lung ventilation is applied to the allograft side. For patients with interstitial pulmonary fibrosis (IPF) it is important to keep in mind that IPF flare can be precipitated by surgery, leading to reduced lung compliance in the native lung.

\section{Fluid management}

Transplanted lungs are liable to develop pulmonary edema due to impaired lymphatic drainage, increased vascular permeability, massive fluid resuscitations or presence of a hyperdynamic right ventricle (9). This reperfusion edema, often referred to as PGD, can be reduced by individualizing resuscitation to maintain a fine balance between preserving adequate cardiac output, urine output and peripheral perfusion while avoiding pulmonary edema. Pulmonary capillary wedge pressure should be kept low $(5-15 \mathrm{mmHg})$, while maintain a mean arterial pressure $>65 \mathrm{mmHg}$ and urine output $>0.5-1 \mathrm{cc} / \mathrm{kg} / \mathrm{h}$. The ideal fluid choice for lung transplant recipients is unclear but crystalloids are reasonable and cost-effective initially as most patients tend be acidotic in the first few hours after surgery. If large amounts of crystalloids are needed (i.e., >1-2 L), colloid transfusions (intravenous albumin, fresh frozen plasma, etc.) can be used. Blood transfusions are usually avoided unless necessary to maintain $\mathrm{Hg}$ levels above $7-8 \mathrm{~g} / \mathrm{dL}$ range.

\section{Sedation and pain control}

Clamshell incision and thoracotomies are notorious for causing significant pain which can limit recovery. Although the ideal combination of sedative and analgesics for pain control is unclear, intravenous (IV) opiates combined with propofol are frequently used especially while the patient is still ventilated. Dexmedetomidine infusion is a good alternative as it causes less respiratory depression, controls agitation and anxiety, and allows for smoother extubation (10). It also can be used safely after extubation. Other agents such as ketamine, gabapentinoids, acetaminophen, and nonsteroidal anti-inflammatory medications are also effective, especially when patients are extubated and do not require sedation. Cryoanalgesia of the intercostal nerves at the time of surgery, usually from the $3^{\text {rd }}$ to the $7^{\text {th }}$ intercostal space, has been shown to have value in reducing post-thoracotomy pain for non-transplant operations but has not been studied in lung transplant patients. If cryoanalgesia is not performed at the time of surgery, we personally prefer the placement of an epidural catheter for pain control before extubation, provided that the coagulation profile is normal. Otherwise, in cases where the patient needs anticoagulation or has an abnormal coagulation profile, paravertebral blocks can be useful adjuncts for pain control.

\section{Initiation of Immunosuppression}

Immunosuppression regimens in lung transplantation are categorized as: induction, maintenance and treatment of rejection. Lung tissue is considered highly immunogenic and most rejections happen in the first 6 months. Most protocols utilize high intensity immunosuppression therapy in the initial post-operative period and decrease it over the first year to the lowest level that prevents graft rejection. Induction therapy aims at abolishing the $\mathrm{T}$-cell response to the transplanted graft and allowing the use of less toxic doses of immunosuppressive agents during the maintenance phase (11). According to the International Society for Heart and Lung Transplantation (ISHLT) registry, the use of induction therapy for lung transplantation increased from $43 \%$ in 2005 to $81 \%$ in 2018 (12). Commonly used induction agents are high dose glucocorticoids (methylprednisolone 500-1,000 mg) intraoperatively before perfusing the allograft, anti-thymocyte globulins (eATG, rATG) with antibodies against human T-cell, or Interleukin-2 (IL-2) receptor antagonist (Basiliximab) which inhibits IL-2 mediated proliferation and differentiation of T-cells (13). Maintenance therapy usually consist triple agents: glucocorticosteroids (prednisone), a calcineurin inhibitor (tacrolimus or cyclosporine) and a nucleotideblocking agent (mycophenolate mofetil or azathioprine). The mTOR inhibitors (sirolimus or everolimus) are used as replacement for calcineurin inhibitors or nucleotide blocking agents. In the 2019 registry report from the ISHLT, $62 \%$ of all lung transplant recipients were on a combination of tacrolimus, mycophenolate, and prednisone a year after lung transplantation (14). Calcineurin inhibitors will require continuous monitoring of blood levels so as to achieve a high mean blood level over time. Minimizing the variations in cyclosporine blood level is associated with reduction in allograft rejection (15). In cases of severe infection, we tend to reduce immunosuppression by holding 
off the nucleotide-blocking agent (mycophenolate). If further reduction is needed, we lower the calcineurin target level but avoid holding immunosuppression altogether due to the risk of rejection.

\section{Infection prophylaxis}

Infectious complications are significant causes of morbidity and mortality, and are currently the $2^{\text {nd }}$ most common cause of death after graft failure (12). Post-operative prevention should include antibacterial, antifungal and antiviral prophylaxis. Antibacterial prophylaxis varies between centers but mostly it should include Gram +ve and Gram ve antibiotic coverage started at the induction of anesthesia and discontinued after 72 hours if all intraoperative cultures are negative and there no signs of an ongoing infection. Viral infections are uncommon in the immediate postoperative period, but the impact on short-term graft function is significant with cytomegalovirus (CMV) infections (16). Prophylaxis is started in immediately after surgery regardless of the recipient and donor CMV status. Intravenous ganciclovir is started first and transitioned to valganciclovir once the patient can tolerate oral intake. The duration of anti-viral therapy is usually 6 months but for patients with donor-positive CMV immunoglobulin, lifelong prophylaxis may be warranted.

Invasive fungal infections are associated with higher mortality (17). The more common causative species are Candida and Aspergillus. Antifungal protocols vary between centers but most use azole antifungals for 3 to 6 months after transplantation. Solid organ transplant recipients are susceptible to Pneumocystis jirovecii pneumonia infection, which is associated with high mortality rates. The first choice for prophylaxis is trimethoprim/sulfamethoxazole administered post-operatively once the renal function is stable and for duration of usually 6 months to 1 year. Pentamidine or dapsone are other alternative drugs for $P$. jirovecii infection.

\section{Additional post-operative considerations}

\section{Perioperative utilization of ECMO}

ECMO utilization in lung transplantation has gained substantial momentum in the last decade. It can be used in the preoperative period as a bridge to transplant, intraoperatively to facilitate technical optimization and hemodynamic stability during the procedure with the option of post-operative prolongation in certain situations, and to treat postoperative PGD $(18,19)$. Prolongation of intraoperative Venoarterial ECMO through the early postoperative recovery is beneficial for patients with pulmonary hypertension, as it diverts part of the cardiac output away from the lungs, thus minimizing the shear stress that the pulmonary vasculature is subjected to by the hyperdynamic right ventricle $(18,19)$. It is important to maintain PA pulsatility while on VA ECMO, since this is the main blood supply to the bronchial structures in the allograft.

\section{Venous thromboembolism}

Lung transplant recipients are at moderate risk for venous thromboembolism (20). Because of the absence of collateral bronchial circulation, pulmonary embolism can be a serious complication, leading to permanent graft compromise or mortality. All patients should receive venous thromboembolism (VTE) prophylaxis, usually in the form of subcutaneous unfractionated heparin until hospital discharge. Systemic therapeutic anticoagulation may be used in patients with a previous history or newly documented VTE, along with oral anticoagulation after discharge.

\section{Atrial arrhythmias}

Atrial arrhythmias such as atrial fibrillation and flutter are common after any thoracic operations involving the lungs or heart, possibly due to the manipulation of the PVs and the left atrium (20). Prompt treatment is warranted, usually with a calcium-channel blocker, beta blocker or amiodarone, although this is controversial in lung transplant recipients (21). When associated with hemodynamic instability, electrocardioversion should be considered. Irregular atrial arrhythmias persisting for more than 48 hours may warrant anticoagulation.

\section{Nutritional support and stress ulcer prophylaxis}

Most lung transplant patients suffer from gastroesophageal reflux, gut dysmotility or delayed emptying. This could be either a manifestation of a preexisting condition or new due to medications or vagus nerve injury during surgery. Recurrent laryngeal nerve injury may occur especially on the left side and leads to an increased risk of aspiration. Stress ulcer prophylaxis with proton-pump inhibitors of $\mathrm{H}_{2}$ blockers is warranted in all patients. Speech therapy is recommended before commencing oral intake to avoid aspiration. In cases of severe dysmotility or delayed emptying, enteric feeding access is preferred. The routine use of $\mu$-receptor antagonists (e.g., methylnaltrexone) in 
patients receiving opioid narcotics has not been proven to prevent the development of post-op ileus and should be used selectively.

\section{Chest tube management}

There is no current evidence regarding chest tube management in lung transplant recipients. Management of chest tubes varies among institutions and even among surgeons in the same institution. Because of the high output and risk of hemothorax after transplant, the use of multiple chest tubes ranging from 2-3 in each pleural space appears to be a standard practice. The use of chest tube suction vs. water seal has not been studied in lung transplant patients, but does not appear to make a difference in non-transplant lung surgery. However, in our institution chest tubes are placed to suction immediately after surgery because of the high-volume output, greater chance of post-op bleeding and hemothorax formation, all of which can lead to lung compression and atelectasis if not adequately drained. Chest tubes can be removed gradually once output from the entire pleural space consistently decreases and there is no obvious air leak or pneumothorax. There is no data currently to guide the upper limit of drainage before removing tubes. Similarly, the practice of routinely clamping chest tubes prior to removal is not supported by literature but may help with decision-making in some cases where there is a risk of developing a pneumothorax after chest tube removal.

\section{Diagnosis and management of acute complications}

\section{Medical complications}

\section{PGD}

PGD is the result of a multi-factorial injury process to the allograft that occurs during the first 72 hours after transplant. Other terms for PGD include ischemia-reperfusion injury, early graft dysfunction and reimplantation edema. PGD is defined as hypoxemia in the first 72 hours associated with diffuse radiographic pulmonary opacities of unknown cause, with the typical histologic finding of diffuse alveolar damage. Up to $30 \%$ of immediate post-operative mortality is attributed to PGD (22), and it is also associated with poor outcomes including prolonged ICU stay, hospitalization, bronchiolitis obliterans and chronic rejection $(23,24)$.

Multiple predisposing factors for PGD have been identified, some of which are reversible (25). These include:

(I) Donor factors: (i) Age $<21$ or $>45$ years

(ii) Female gender

(iii) Smoker

(iv) African-American origin

(II) Recipient factors:

(i) Overweight and obesity BMI $>25$

(ii) Preoperative sarcoidosis or pulmonary hypertension

(iii) Single lung transplant

(III) Intraoperative factors:

(i) Increased $\mathrm{FiO}_{2}$ during allograft reperfusion (26)

(ii) Use of cardiopulmonary bypass

(iii) Administration of blood products during surgery

\section{Management of PGD}

For PGD grade 2 or less, conventional management is in the form of protective lung ventilation (low tidal volume, high PEEP), meticulous fluid balance, and inhaled NO. For severe cases (PGD 3) or those unresponsive to conventional management, ECMO should be initiated and most patients will improve on Venovenous (VV)-ECMO (27). VA-ECMO is reserved for patients with hemodynamic instability or pulmonary hypertension. It is important to note that VAECMO will shunt the oxygenated blood from the lungs, aggravating bronchial ischemia and therefore it is crucial to maintain pulsatile pulmonary arterial flow.

\section{Acute allograft rejection}

Acute rejection in lung transplant is relatively common especially during the first year. ISHLT registry data showed a prevalence of $28 \%$ for acute rejection (28), which is an important predisposing factor in the development of bronchiolitis obliterans syndrome (BOS). Acute rejection is further categorized as acute cellular rejection or acute antibody-mediated rejection (AMR). Clinical presentation is nonspecific and includes cough, fever, dyspnea, malaise, pleural effusion. CT scan of the chest may show groundglass appearance with lower lobe predominance; such radiographic findings along with symptoms should lead to a high index of suspicion for acute rejection. Transbronchial biopsy is the most important diagnostic tool: acute cellular rejection (ACR) will typically show perivascular and peribronchial lymphocytic infiltrates, while AMR is associated with less specific pathologic findingslung biopsy may show complement $4 \mathrm{~d}(\mathrm{C} 4 \mathrm{~d})$ peritubular capillary staining (29). Treatment of acute rejection usually consists of pulse steroids for 3 days and optimization 
of immunosuppression. AMR is less responsive to steroids, hence additional options such as plasmapheresis, intravenous immunoglobulin (IVIG) infusion; anti-CD20 monoclonal antibodies are part of the treatment protocol.

Hyperacute rejection, although very rare with current human leukocyte antigen (HLA) screening methods, presents within minutes to hours after lung perfusion and is due to pre-formed donor specific antibodies (DSA). Severe allograft dysfunction, hemodynamic instability, coagulopathy, hemorrhagic pulmonary edema and severe gas exchange impairment are characteristic features. Lung biopsy may not be feasible or safe and the diagnosis should be presumptive based on the clinical presentation and positive cross-match. Hyperacute rejection protocol consists of plasmapheresis, antithymocyte globulin treatment and cyclophosphamide in addition to high-dose steroids (30) but salvage of the graft and the patient can be very challenging. Urgent retransplantation should be considered while treatment is being continued.

\section{Surgical complications}

\section{Vascular complications}

During transplantation the bronchial anastomosis is performed first, followed by the two major vascular anastomoses. The pulmonary venous anastomosis is created between the donor left atrial cuff and the confluence of the recipient PVs. The donor main PA is anastomosed to the ipsilateral main PA or to an equally sized large branch. Both anastomoses are susceptible to complications which can be prevented with proper surgical techniques. Vascular complications are broadly classified into:

(I) Anastomotic leaks

(II) Anastomotic stenosis

(III) Non-anastomotic obstruction

\section{Anastomotic leaks}

The vascular tissues of the transplant recipient are quite fragile due to chronic illness and steroid use. Anastomotic leaks (bleeding) are usually identified intra-operatively and can often be repaired with a horizontal mattress or figureof-eight suture. Pledgets made from the donor pericardium may help in reinforcement of fragile tissue and helps to avoid the use of synthetic material (e.g., felt or Teflon) in the immunocompromised patients. It is preferable that repair sutures are placed with clamps released and the vessels distended to avoid suturing the back wall, unless bleeding impairs proper visualization. Revision of the entire anastomosis is rarely necessary.
Anastomotic bleeding can also present during the immediate post-operative period as increased sanguineous chest tube output, hemodynamic instability and drop in the hemoglobin levels. Management will depend on the presence of shock, hemodynamic stability, coagulation profile, response to resuscitation and the degree of lung compression by any hemothorax. Chest X-ray, CT scans or pleural ultrasound can help detect significant hemothorax and may also reveal occult bleeding when chest tubes are clogged. Immediate exploration is warranted in patients with massive bleeding, hemodynamic instability or significant hemothorax. During re-exploration, lung isolation with double-lumen endotracheal tube will significantly improve visualization when feasible. Otherwise, intermittent hypoventilation or apnea can be performed to help with exposure. Occasionally, cardiopulmonary bypass or VA-ECMO may be necessary for difficult exposures or complicated anastomotic repairs.

\section{Anastomotic stenosis}

Anastomotic stenosis occurs in $<5 \%$ of lung transplant procedures (31). While both arterial and venous anastomoses are susceptible, venous stenosis is far more devastating to the graft and the patient. Attention to details is the key in preventing this complication, which is usually a technical issue. Size-matching of the donor and recipient vascular lumina is important to avoid hour-glass shaped narrowing of the anastomosis. Techniques to overcome significant size-mismatch include trimming excess edges on the larger vessel, enlarging the smaller one with spatulation, pericardial patch angioplasty or division of the common wall between smaller vessels to create a common orifice (32-34). Trimming the donor vessels close to the lobar origins may risk stenosis when suturing. Purse-string effects that occur during suture tying can be avoided by releasing the vascular clamps before tying and by not pulling too tightly on the sutures. Another maneuver is to lock the suture once each on the anterior wall and the posterior portion of the anastomosis during suturing.

Severe venous stenosis can present as pulmonary congestion and edema after reperfusion of the transplanted lung. The lung will appear dusky, non-elastic, and feel heavy and firm to touch. In cases of mild to moderate stenosis, findings may appear hours to days later with persistent radiographic opacification of the affected lung, pulmonary edema and excessive clear chest tube drainage (32).

PA stenosis can present with pulmonary hypertension, hypoxia, difficulty in weaning off the ventilator and pulmonary thrombus formation due to turbulent flow in 
the stenosed segment. TEE is an excellent investigative tool to diagnose PVs stenosis and assess the degree of stenosis by measuring the pressure gradients across the anastomosis (35), although the left lower PV can be difficult to visualize. Non-invasive CT pulmonary angiography is commonly used to assess for vascular stenosis. Traditional pulmonary angiography can be diagnostic and therapeutic but is more invasive. Lung ventilation-perfusion scans can show the area of perfusion defects.

Vascular stenosis should be repaired promptly when detected intraoperatively. Post-operative arterial stenosis can often be corrected by percutaneous angioplasty and stenting (36). Venous stenosis is more difficult to treat nonsurgically and usually requires urgent surgical exploration and anastomosis revision. When revising the PV anastomosis, heparin should be administered, ventilation stopped to the affected side and the PA clamped. After the lung is well drained, the $\mathrm{PV}$ is clamped medial to the anastomosis (toward the heart) prior to the repair.

\section{Non-anastomotic obstructions}

Non-anastomotic obstructions may be due to kinking of the pulmonary vessel, external compression, or intraluminal thrombus (32). Since the vascular anastomoses are constructed with the lungs deflated and the proximal vessels clamped, the optimal vessel length may be difficult to determine. Any redundancy, twisting or kinking of the pulmonary vessels should be addressed immediately after flow restoration. External vascular compression can result from large clots, packing of hemostatic materials or excessive wrapping with pericardial flap, all of which should be addressed when identified.

Intraluminal thrombus formation can happen during the organ procurement due to inadequate flushing or perfusion techniques. These thrombi are liable to dislodgement after lung perfusion and can result in varying degree of lung infarction. If discovered during the back-table organ preparation, they can be removed by a Fogarty catheter and repetition of antegrade and retrograde flushing with lung protective perfusate (37).

Pulmonary venous thrombi develop in $15 \%$ of patients during the early post-operative period, with a mortality rate of $38 \%$ in the first 3 months (38). Allograft failure and systemic embolization are the main concerns of venous thrombi. Systemic antithrombotic therapy should be initiated for moderate or large venous thrombi causing narrowing on TEE, and followed by oral anticoagulation. Smaller thrombi, especially those with minimal elevation of peak pulmonary venous velocity can be observed (38).
Severe venous obstruction from thrombi may require thrombus extraction and revision of the anastomosis, although the mortality is high (35).

\section{Airway complications}

Normal human lungs have two sources of blood supply: the systemic (bronchial artery) and the pulmonary circulation. Transplantation deprives the lungs of the systemic (bronchial) supply, making the pulmonary (deoxygenated) circulation the main source for bronchial blood supply through low pressure retrograde collaterals (39). Ischemia of the donor bronchus is universally present in all transplants but is rarely clinically significant when mild to moderate.

The most common acute ( $<1$ month) airway complications include bronchial dehiscence and fistulas. Other types which can happen later include stenosis (the most common type), endobronchial granulomas, bronchomalacia and endobronchial infections. Pre-operative risk factors include pulmonary infections, inadequate organ preservation, donorrecipient size mismatch involving taller recipient, and prolonged donor ventilation before procurement $(39,40)$. Post-operatively intense immunosuppression, rejection and prolonged ventilation can impair bronchial healing. In recent years better patient selection, surgical techniques, immunosuppression and lung preservation have led to lower incidence of airway complications (39).

Adherence to proper surgical techniques remains the most important factor in reducing bronchial ischemia. Excessive trimming and denuding of peribronchial tissues should be avoided in both donor and recipient. Lymph nodes around the hilum do not require complete resection and extensive lymphadenectomy may reduce bronchial blood supply. The donor bronchus is resected to approximately one or two rings above the secondary carina to reduce the length of the bronchial segment at risk for ischemia (41). Various anastomotic techniques have been tried in an effort to improve bronchial healing; the two techniques mostly used are end-to-end anastomosis and telescoping. Telescoping has fallen out of favor recently due to multiple reports showing increased incidence of airway complications (42). Techniques used in the early transplant era to improve blood supply to the bronchial anastomosis such as wrapping with a pedicled omental flap and bronchial arterial revascularization are no longer advocated due to their complexity and the lower incidence of airway complications in recent years $(43,44)$.

\section{Debiscence}

Post-transplant bronchoscopy typically reveals some degree 
of perianastomotic mucosal necrosis which is usually partial and resolves spontaneously (45). Full-thickness necrosis may occur in $1.5 \%$ to $10 \%$ of anastomoses and can lead to varying degrees of dehiscence. This can significantly increase the risk of pleural space contamination, empyema and sepsis $(42,46)$. Delayed pneumothorax or air leak in the chest tube should prompt investigation with bronchoscopy. High-resolution CT scan is sensitive in detecting dehiscence and may show peribronchial extra-luminal air, air-fluid collection or loss of bronchial wall continuity (47). Small $(<4 \mathrm{~mm})$ bronchial defects usually heal spontaneously. Endobronchial stenting with self-expandable metallic or silicon stents that promote growth of granulation tissues is the treatment of choice for most airway dehiscences (48). Larger airway disruptions which are refractory or nonamenable to stenting are difficult to manage due to the persistent impaired blood supply even after anastomosis reconstruction. In this situation, the use of a vascularized pedicled flap such as intercostal muscle, pericardial fat or omental flap is crucial in augmenting the anastomosis (49).

\section{Fistulas}

Bronchial dehiscence may rarely lead to fistulas connecting the airway to the pleural space, a blood vessel, mediastinum or even the heart. Broncho-vascular fistulas can initially present with small amount of blood-stained sputum, 'herald bleeding', which can be followed by massive hemoptysis. Fistulas to the left atrium can cause air embolism. Minor hemoptysis should be investigated with bronchoscopy and pulmonary angiography, while massive bleeding is a surgical emergency (50), and requires immediate intubation, isolation of the affected side, and positioning the patient in a lateral decubitus with the affected side in a dependent position to avoid flooding the other lung. Cardiopulmonary bypass may be needed to stabilize the patient and aid in fistula exposure and repair.

Management of non-vascular fistulas will depend on the fistula size and clinical condition of the patient. Goal of the treatment is to eradicate infection, prevent cross contamination of the other lung and stabilize the patient. Treatment options can vary from conservative approach with endoscopic therapy (51), antibiotics and thoracostomy tubes to more complex procedures like the Eloesser window, a modified Clagett procedure, lobectomy or even pneumonectomy (50). Any surgical repair of bronchial fistula should include a well vascularized interpositional soft tissue flap.

\section{Strictures}

Stenosis is the most common post-transplant delayed airway complication due to ischemia and occurs in up to $32 \%$ of anastomoses $(42,52)$. Strictures develop 2 to 10 months after transplantation and present with reduced FEV1 which is associated with a lower survival. The majority $(>85 \%)$ of strictures involves the anastomosis, but in a small number of cases non-anastomotic segmental stenosis can occur with the bronchus intermedius being mostly involved (53). Management is usually with bronchoscopic dilation, with or without stenting.

\section{Air leaks}

Air leaks are relatively common after lung transplant. Possible sources are from the bronchial anastomosis or lung parenchyma, especially if adhesiolysis is needed during donor procurement. Bronchial anastomoses should be checked for leaks after reperfusion by performing a Valsalva maneuver to a pressure of $30 \mathrm{~cm} \mathrm{H}_{2} \mathrm{O}$ with the bronchus immersed under normal saline. Parenchymal tears on the lung surface can be repaired with a running $4 / 0$ monofilament suture or by stapled wedge resection of the disrupted area. Most air leaks in the immediate post-operative period resolve spontaneously and can be managed by the chest tubes (54). Delayed appearance of air leaks can be a sign of bronchial dehiscence and need further investigation.

\section{Phrenic nerve injury}

The phrenic nerve runs in close proximity to the lung hilum and is at an increased risk of injury during dissection, either directly or by thermal injury. Patients with severe intrathoracic adhesions or undergoing reoperative surgery have the highest risk of phrenic nerve injury due to difficulty in identification.

Phrenic nerve injury may lead to temporary or permanent diaphragmatic paralysis. Evaluation can be done by a sniff test with fluoroscopy or ultrasound. Although long term sequelae are not significant, diaphragmatic paralysis may lead to prolonged ventilation and ICU stay (55). Diaphragmatic plication can be done for severe permanent paralysis, although current data does not show a significant benefit over observation.

\section{Post-operative hemorrhage}

Post-operative bleeding is reported in up to $18 \%$ of lung recipients and is considered a major predictor of morbidity and mortality (56). The pleural space is the most common site for bleeding; other sources include the vascular anastomoses, the mediastinum, and the chest wall or subcutaneous spaces. The main risk factors for post- 
operative bleeding are the presence of extensive adhesions and cardiopulmonary bypass.

Intrathoracic hematomas should be explored since the collected blood clots may become infected and lead to wound abscesses and empyema. Undrained moderate to large hematomas can affect the expansion of the lung and cause persist collapse and impaired gas exchange. Meticulous hemostasis before chest closure can reduce the incidence of post-operative hematomas. Various hemostatic agents and devices including argon beam coagulators can aid in controlling the bleeding from raw surfaces and chest wall cavity. The use of intrapleurally administered tissue plasminogen activator ( $\mathrm{tPA}$ ) to lyse the hematoma may increase the chance of successful chest tube drainage and avoid reoperation but this has not been studied in the transplant population. Intrapleural tPA should be used only if active bleeding has been ruled out since there is a risk of increased bleeding if the fresh clot tamponading a bleeding source is dissolved.

\section{Pleural space complications}

Pleural space conditions that require intervention are seen in up to $22 \%$ of lung transplant procedures (54). Conditions like pleural effusion and pneumothorax are commonly seen, whereas empyema and chylothorax are much less common (57). Symptomatic pneumothorax or when $>5 \%$ of the hemithorax volume is managed by percutaneous pigtail catheter placement under ultrasound or CT guidance. A small, non-expanding pneumothorax can be safely observed as this may be due to smaller-sized donor lungs that have not filled the pleural space entirely. Pleural effusions are managed according to size and complexity; smaller effusions without infection or pulmonary compression can be observed (54). Large non-loculated effusions can be drained with percutaneous catheters or chest tubes. For loculated effusions, tPA in combination with deoxyribonuclease (dornase alfa) can be instilled as a solution through a chest tube or pigtail catheter to help break down the loculations (58). This regimen can also be used in empyema although a lower threshold for surgical exploration is preferred. Thoracoscopy is often used for explorations and washouts, but occasionally an open thoracotomy approach is needed for more dense and complicated effusions. Pulmonary decortication may be required for entrapped lungs not responding to tPA and dornase.

\section{Donor-recipient size mismatch}

Donor-recipient size mismatch is frequently encountered in lung transplantation with either oversized or undersized donor lungs. Donor lung oversizing commonly occurs in male donor to female recipient, or when the recipient lung pathology causes reduced lung capacity, such as in idiopathic pulmonary fibrosis, cystic fibrosis and previous recipient lobectomy. Undersizing is less common and occurs especially when recipients have large chest cavities, e.g., in long standing emphysema.

Donor-recipient oversizing is often managed by simple stapled wedge resection of part(s) of the implanted lung, often the right middle lobe or the lingula of the left upper lobe. Wedge resection carries a low risk of air leaks, as opposing pleura will seal the resected surfaces. We typically use reinforced collagen-strip buttressing of the staplers to reduce the risk of staple line disruption. Other options for downsizing are lobar transplants or wedge resection of the upper lobes, compressed parts of the basal segments of the lower lobes or anatomic lobectomy during back table preparation (59). Occasionally primary closure of the chest in lung transplant may not be possible due to severe oversizing or graft swelling from massive resuscitation or severe PGD. Closure attempts in such situations may result in thoracic compartment syndrome manifesting as hemodynamic instability and pulmonary compromise. This is managed by leaving the chest open with airtight sterile dressing and adequate chest drains. Chest closure can be attempted in a few days later when the graft edema resolves.

Undersized grafts can lead to persistent pleural space and recurrent effusions. In addition, long-term outcomes and survival are adversely affected by undersizing of the lungs (60). Management of undersized lungs is usually directed at dealing with the symptoms and clinical findings as they manifest. Unfortunately, there is no way to increase the size of the graft once implanted and retransplantation with properly sized grafts is the only permanent solution to this problem.

\section{Complications during single lung transplantation}

Single lung transplantation can cause certain complications that are not typically seen with bilateral transplantation. It is important for the transplanting team to be aware of these potential complications which can often be prevented by proper identification of the risk factors. In emphysema, preferential ventilation and over-expansion of the native lung can lead to mediastinal shift and graft compression. In severe cases of hyperinflation, lung reduction surgery or lobectomy of the non-transplanted 
lung maybe necessary to reduce graft compromise (61). In fibrotic lung disease, the attenuated vascular bed and increased pulmonary microcapillary vascular resistance within the native lung can divert disproportionately more blood to the transplanted side. This can cause hyperperfusion, pulmonary edema, graft injury and right heart failure. Careful preoperative evaluation to rule out moderate or severe pulmonary hypertension is important to prevent this complication. Severe differential perfusion that compromises graft function can be managed by VA ECMO to stabilize the patient and salvage the graft while considering listing for emergent re-transplantation. If the graft is salvageable, single transplantation of the non-transplanted side will suffice; otherwise bilateral retransplantation will be necessary. In long-term surviving single lung recipients with IPF, the native lung may shrink overtime and can cause chronic relentless cough that may necessitate lobectomy (62).

\section{Acknowledgments}

Funding: None.

\section{Footnote}

Provenance and Peer Review: This article was commissioned by the Guest Editor (George Makdisi) for the series "Lung Transplant: Current Status and Challenges" published in Current Challenges in Thoracic Surgery. The article has undergone external peer review.

Conflicts of Interest: Both authors have completed the ICMJE uniform disclosure form (available at https://ccts.amegroups. com/article/view/10.21037/ccts-20-182/coif). The series "Lung Transplant: Current Status and Challenges" was commissioned by the editorial office without any funding or sponsorship. The authors have no other conflicts of interest to declare.

Ethical Statement: The authors are accountable for all aspects of the work in ensuring that questions related to the accuracy or integrity of any part of the work are appropriately investigated and resolved.

Open Access Statement: This is an Open Access article distributed in accordance with the Creative Commons Attribution-NonCommercial-NoDerivs 4.0 International License (CC BY-NC-ND 4.0), which permits the non- commercial replication and distribution of the article with the strict proviso that no changes or edits are made and the original work is properly cited (including links to both the formal publication through the relevant DOI and the license). See: https://creativecommons.org/licenses/by-nc-nd/4.0/.

\section{References}

1. Yusen RD, Edwards LB, Kucheryavaya AY, et al. The Registry of the International Society for Heart and Lung Transplantation: Thirty-second Official Adult Lung and Heart-Lung Transplantation Report--2015; Focus Theme: Early Graft Failure. J Heart Lung Transplant 2015;34:1264-77.

2. O'Horo JC, Maki DG, Krupp AE, et al. Arterial catheters as a source of bloodstream infection: a systematic review and meta-analysis. Crit Care Med 2014;42:1334-9.

3. Chenoweth C, Saint S. Preventing catheter-associated urinary tract infections in the intensive care unit. Crit Care Clin 2013;29:19-32.

4. Rassler B. Contribution of $\alpha$ - and $\beta$-Adrenergic Mechanisms to the Development of Pulmonary Edema. Scientifica (Cairo) 2012;2012:829504.

5. Beer A, Reed RM, Bölükbas S, et al. Mechanical ventilation after lung transplantation. An international survey of practices and preferences. Ann Am Thorac Soc 2014;11:546-53.

6. Miyoshi R, Chen-Yoshikawa TF, Hamaji M, et al. Effect of early tracheostomy on clinical outcomes in critically ill lung transplant recipients. Gen Thorac Cardiovasc Surg 2018;66:529-36.

7. Yonan NA, el-Gamel A, Egan J, et al. Single lung transplantation for emphysema: predictors for native lung hyperinflation. J Heart Lung Transplant 1998;17:192-201.

8. Eberlein M, Reed RM, Bolukbas S, et al. Lung size mismatch and primary graft dysfunction after bilateral lung transplantation. J Heart Lung Transplant 2015;34:233-40.

9. Geube MA, Perez-Protto SE, McGrath TL, et al. Increased Intraoperative Fluid Administration Is Associated with Severe Primary Graft Dysfunction After Lung Transplantation. Anesth Analg 2016;122:1081-8.

10. Carollo DS, Nossaman BD, Ramadhyani U. Dexmedetomidine: a review of clinical applications. Curr Opin Anaesthesiol 2008;21:457-61.

11. Yeung JC, Keshavjee S. Overview of clinical lung transplantation. Cold Spring Harb Perspect Med 
2014;4:a015628.

12. Chambers DC, Yusen RD, Cherikh WS, et al. The Registry of the International Society for Heart and Lung Transplantation: Thirty-fourth Adult Lung And HeartLung Transplantation Report-2017; Focus Theme: Allograft ischemic time. J Heart Lung Transplant 2017;36:1047-59.

13. Chandrashekaran S, Crow Pharm SA, Shah SZ, et al. Immunosuppression for Lung Transplantation: Current and Future. Curr Transplant Rep 2018;5:212-9.

14. Chambers DC, Cherikh WS, Harhay MO, et al. The International Thoracic Organ Transplant Registry of the International Society for Heart and Lung Transplantation: Thirty-sixth adult lung and heart-lung transplantation Report-2019; Focus theme: Donor and recipient size match. J Heart Lung Transplant 2019;38:1042-55.

15. Pollock-Barziv SM, Finkelstein Y, Manlhiot C, et al. Variability in tacrolimus blood levels increases the risk of late rejection and graft loss after solid organ transplantation in older children. Pediatr Transplant 2010;14:968-75.

16. Zamora MR, Nicolls MR, Hodges TN, et al. Following universal prophylaxis with intravenous ganciclovir and cytomegalovirus immune globulin, valganciclovir is safe and effective for prevention of CMV infection following lung transplantation. Am J Transplant 2004;4:1635-42.

17. Chong PP, Kennedy CC, Hathcock MA, et al. Epidemiology of invasive fungal infections in lung transplant recipients on long-term azole antifungal prophylaxis. Clin Transplant 2015;29:311-8.

18. Ius F, Tudorache I, Warnecke G. Extracorporeal support, during and after lung transplantation: the history of an idea. J Thorac Dis 2018;10:5131-48.

19. Hoetzenecker K, Schwarz S, Muckenhuber M, et al. Intraoperative extracorporeal membrane oxygenation and the possibility of postoperative prolongation improve survival in bilateral lung transplantation. J Thorac Cardiovasc Surg 2018;155:2193-206.e3.

20. Dizon JM, Chen K, Bacchetta M, et al. A comparison of atrial arrhythmias after heart or double-lung transplantation at a single center: insights into the mechanism of post-operative atrial fibrillation. J Am Coll Cardiol 2009;54:2043-8.

21. Isiadinso I, Meshkov AB, Gaughan J, et al. Atrial arrhythmias after lung and heart-lung transplant: effects on short-term mortality and the influence of amiodarone. J Heart Lung Transplant 2011;30:37-44.

22. Christie JD, Sager JS, Kimmel SE, et al. Impact of primary graft failure on outcomes following lung transplantation. Chest 2005;127:161-5.

23. Prekker ME, Nath DS, Walker AR, et al. Validation of the proposed International Society for Heart and Lung Transplantation grading system for primary graft dysfunction after lung transplantation. J Heart Lung Transplant 2006;25:371-8.

24. Whitson BA, Prekker ME, Herrington CS, et al. Primary graft dysfunction and long-term pulmonary function after lung transplantation. J Heart Lung Transplant 2007;26:1004-11.

25. Diamond JM, Lee JC, Kawut SM, et al. Clinical risk factors for primary graft dysfunction after lung transplantation. Am J Respir Crit Care Med 2013;187:527-34.

26. Shaver CM, Ware LB. Primary graft dysfunction: pathophysiology to guide new preventive therapies. Expert Rev Respir Med 2017;11:119-28.

27. Wigfield CH, Lindsey JD, Steffens TG, et al. Early institution of extracorporeal membrane oxygenation for primary graft dysfunction after lung transplantation improves outcome. J Heart Lung Transplant 2007;26:331-8.

28. Lund LH, Khush KK, Cherikh WS, et al. The Registry of the International Society for Heart and Lung Transplantation: Thirty-fourth Adult Heart Transplantation Report-2017; Focus Theme: Allograft ischemic time. J Heart Lung Transplant 2017;36:1037-46.

29. Levine DJ, Glanville AR, Aboyoun C, et al. Antibodymediated rejection of the lung: A consensus report of the International Society for Heart and Lung Transplantation. J Heart Lung Transplant 2016;35:397-406.

30. Bittner HB, Dunitz J, Hertz M, et al. Hyperacute rejection in single lung transplantation--case report of successful management by means of plasmapheresis and antithymocyte globulin treatment. Transplantation 2001;71:649-51.

31. Clark SC, Levine AJ, Hasan A, et al. Vascular complications of lung transplantation. Ann Thorac Surg 1996;61:1079-82.

32. Griffith BP, Magee MJ, Gonzalez IF, et al. Anastomotic pitfalls in lung transplantation. J Thorac Cardiovasc Surg 1994;107:743-53; discussion 53-4.

33. Oto T, Rabinov M, Negri J, et al. Techniques of reconstruction for inadequate donor left atrial cuff in lung transplantation. Ann Thorac Surg 2006;81:1199-204.

34. Belli EV, Landolfo K, Thomas M, et al. Partial anomalous pulmonary venous return in a lung transplant recipient. 
Ann Thorac Surg 2013;95:1104-6.

35. González-Fernández C, González-Castro A, RodríguezBorregán JC, et al. Pulmonary venous obstruction after lung transplantation. Diagnostic advantages of transesophageal echocardiography. Clin Transplant 2009;23:975-80.

36. Grubstein A, Atar E, Litvin S, et al. Angioplasty using covered stents in five patients with symptomatic pulmonary artery stenosis after single-lung transplantation. Cardiovasc Intervent Radiol 2014;37:686-90.

37. de Perrot M, Keshavjee S. Lung preservation. Semin Thorac Cardiovasc Surg 2004;16:300-8.

38. Schulman LL, Anandarangam T, Leibowitz DW, et al. Four-year prospective study of pulmonary venous thrombosis after lung transplantation. J Am Soc Echocardiogr 2001;14:806-12.

39. Santacruz JF, Mehta AC. Airway complications and management after lung transplantation: ischemia, dehiscence, and stenosis. Proc Am Thorac Soc 2009;6:79-93.

40. Van De Wauwer C, Van Raemdonck D, Verleden GM, et al. Risk factors for airway complications within the first year after lung transplantation. Eur J Cardiothorac Surg 2007;31:703-10.

41. Mulligan MS. Endoscopic management of airway complications after lung transplantation. Chest Surg Clin N Am 2001;11:907-15.

42. Garfein ES, McGregor CC, Galantowicz ME, et al. Deleterious effects of telescoped bronchial anastomosis in single and bilateral lung transplantation. Ann Transplant 2000;5:5-11.

43. Miller JD, DeHoyos A. An evaluation of the role of omentopexy and of early perioperative corticosteroid administration in clinical lung transplantation. The University of Toronto and Washington University Lung Transplant Programs. J Thorac Cardiovasc Surg 1993;105:247-52.

44. Inci I, Weder W. Airway complications after lung transplantation can be avoided without bronchial artery revascularization. Curr Opin Organ Transplant 2010;15:578-81.

45. Alvarez A, Algar J, Santos F, et al. Airway complications after lung transplantation: a review of 151 anastomoses. Eur J Cardiothorac Surg 2001;19:381-7.

46. Murthy SC, Blackstone EH, Gildea TR, et al. Impact of anastomotic airway complications after lung transplantation. Ann Thorac Surg 2007;84:401-9.e4094.

47. Semenkovich JW, Glazer HS, Anderson DC, et al.
Bronchial dehiscence in lung transplantation: CT evaluation. Radiology 1995;194:205-8.

48. Kapoor BS, May B, Panu N, et al. Endobronchial stent placement for the management of airway complications after lung transplantation. J Vasc Interv Radiol 2007;18:629-32.

49. Camargo JJ, Camargo SM, Machuca TN, et al. Surgical maneuvers for the management of bronchial complications in lung transplantation. Eur J Cardiothorac Surg 2008;34:1206-9.

50. Rea F, Marulli G, Loy M, et al. Salvage right pneumonectomy in a patient with bronchial-pulmonary artery fistula after bilateral sequential lung transplantation. J Heart Lung Transplant 2006;25:1383-6.

51. Lois M, Noppen M. Bronchopleural fistulas: an overview of the problem with special focus on endoscopic management. Chest 2005;128:3955-65.

52. Castleberry AW, Worni M, Kuchibhatla M, et al. A comparative analysis of bronchial stricture after lung transplantation in recipients with and without early acute rejection. Ann Thorac Surg 2013;96:1008-17; discussion 17-8.

53. Lari SM, Gonin F, Colchen A. The management of bronchus intermedius complications after lung transplantation: a retrospective study. J Cardiothorac Surg 2012;7:8.

54. Herridge MS, de Hoyos AL, Chaparro C, et al. Pleural complications in lung transplant recipients. J Thorac Cardiovasc Surg 1995;110:22-6.

55. Ferdinande P, Bruyninckx F, Van Raemdonck D, et al. Phrenic nerve dysfunction after heart-lung and lung transplantation. J Heart Lung Transplant 2004;23:105-9.

56. Ferrer J, Roldan J, Roman A, et al. Acute and chronic pleural complications in lung transplantation. J Heart Lung Transplant 2003;22:1217-25.

57. Jacob S, Meneses A, Landolfo K, et al. Incidence, Management, and Outcomes of Chylothorax after Lung Transplantation: A Single-center Experience. Cureus 2019;11:e5190.

58. Ahmed AE, Yacoub TE. Empyema thoracis. Clin Med Insights Circ Respir Pulm Med 2010;4:1-8.

59. Raja S, Murthy SC, Pettersson GB, et al. Managing extreme airway size mismatch in lung transplantation: the "upper lobectomy" technique. Semin Thorac Cardiovasc Surg 2011;23:336-8.

60. Eberlein M, Reed RM. Donor to recipient sizing in thoracic organ transplantation. World J Transplant 2016;6:155-64. 
61. Anderson MB, Kriett JM, Kapelanski DP, et al. Volume reduction surgery in the native lung after single lung transplantation for emphysema. J Heart Lung Transplant 1997;16:752-7.

doi: $10.21037 /$ ccts-20-182

Cite this article as: Haddad O, Thomas M. Postoperative management and acute complications after lung transplantation. Curr Chall Thorac Surg 2021.
62. Elicker BM, Golden JA, Ordovas KG, et al. Progression of native lung fibrosis in lung transplant recipients with idiopathic pulmonary fibrosis. Respir Med 2010;104:426-33. 